\title{
On the faint X-ray sources in the Galactic center region
}

\author{
X.-W. Liu and X.-D. Li \\ Department of Astronomy, Nanjing University, Nanjing 210093, P. R. China \\ email: liuxw@nju.edu.cn, lixd@nju.edu.cn
}

\begin{abstract}
We have performed evolutionary population synthesis calculations to investigate the nature of the faint X-ray sources in the Galactic center region detected by recent Chandra surveys. Our results show that neutron star low-mass X-ray binaries contribute significantly to the observed sources, but the majority of the sources in Wang et al. (2002) survey are still beyond our expectation. We also point out that wind-accreting neutron stars and intermediate polars play a minor role in accounting for the faint X-ray sources in Wang et al. (2002) and Muno et al. (2003) surveys, respectively.
\end{abstract}

Keywords. stellar evolution, compact stars, X-ray binaries.

\section{Introduction}

Recent Chandra observations of the Galactic center region (GCR) have uncovered a population of faint discrete X-ray sources. Wang et al. (2002) have reported hundreds of X-ray sources with luminosities $L_{\mathrm{X}} \sim 10^{33}-10^{35} \mathrm{ergs}^{-1}$ in the GCR. Muno et al. (2003) detected $\sim 2000$ point sources during their 590 ks Chandra observations of the $17^{\prime} \times 17^{\prime}$ field around Sgr A*.

The nature of the faint X-ray sources in the GCR has been investigated by several authors (Pfahl et al. 2002, PRP02; Belczynski \& Taam 2004, BT04). In this paper, we employed the evolutionary population synthesis (EPS) method to calculate the expected numbers and luminosity distributions of various types of X-ray sources in Wang et al. and Muno et al. surveys. We describe the model in $\S 2$. The calculated results are presented in $\S 3$. Our discussion and conclusions are in $\S 4$.

\section{Model}

We have used the EPS code developed by Hurley et al. $(2000,2002)$ to calculate the expected numbers in the Galactic disk for various types of X-ray source populations. Most of our adopted parameters are the same as those described in Hurley et al. (2002) except that we assume that the supernova kick velocity has a Maxwellian distribution with standard deviation $\sigma=265 \mathrm{kms}^{-1}$ (Hobbs et al. 2005).

\subsection{Physical Models for X-ray Sources}

\subsubsection{Wind-fed neutron stars (WNSs)}

A WNS is a neutron star (NS) accreting wind material from an intermediate- or highmass un-evolved companion (PRP02). We consider the total spin evolution of NSs including the ejector, propeller and accretor stages (Davies \& Pringle 1981; Ikhsanov 2001). The parameters of the natal NSs are set as follows. The initial spin periods $P$ and magnetic fields $B$ are chosen so that $\log P$ and $\log B$ are distributed normally with a mean of -2.3 and 12.5 respectively with a standard deviation of 0.3 . 


\subsubsection{Intermediate polars (IPs)}

Muno et al. (2004) suggested that the majority of the GC sources are IPs. To examine this idea, we include cataclysmic variables in our calculations, and set $5 \%$ of them are IPs (Kube et al. 2003).

\subsubsection{Low-mass X-ray binaries (LMXBs)}

This type of systems contains NSs or black holes (BHs) accreting from low-mass companions that overfill their Roche lobes. Most of LMXBs are transients, with the majority of their time spent in quiescence with $L_{\mathrm{X}}<10^{34} \mathrm{ergs}^{-1}$ (van Paradijs 1996). We have adopted the theoretical model of Menou et al. (1999) for X-ray emission of quiescent LMXBs. Both normal star and white dwarf (WD) donors are included in our simulations. Apart from core collapse SNe, the code also includes the formation of NSs via accretion-induced collapse of massive WDs.

\subsubsection{Rotation-powered pulsars}

In the ejector stage, the spin-down of NSs is governed by the canonical rotationpowered pulsar mechanism. There appears to be a strong correlation between the rate of rotational energy loss $\dot{E}$ and their X-ray luminosities $L_{\mathrm{X}}$ (Seward \& Wang 1988). We use the $L_{\mathrm{X}}-\dot{E}$ correlation in Possenti et al. (2002) to estimate the $2-10 \mathrm{keV}$ X-ray luminosities of pulsars.

\subsubsection{Massive stars with strong winds}

The X-ray emission in massive stars is generated in the lower layers of the strong winds, where the plasma is heated in shocks. For single and binary massive stars, we calculate their X-ray luminosities based on the empirical relations in Chelbowski \& Garmany (1991) and Portegies Zwart et al. (2002a), respectively.

\subsection{Luminosity correction}

For WNSs, IPs, and quiescent LMXBs, we assume their 2-10 keV luminosities to be half the calculated bolometric luminosities. No correction is needed for rotation powered pulsars. For massive stars with strong winds the previously estimated luminosities are in the $0.5-3.5 \mathrm{keV}$ band, we choose the correction factor 0.1 for the $2-10 \mathrm{keV}$ luminosities. For disk accreting sources (i.e. IP and LMXBs), the geometric effect of the disks (Zhang 2005 ) is also taken into account for the apparent X-ray luminosities.

\subsection{Expected Numbers}

The size of Wang et al. field contains $\leqslant 1 \%$ of the total Galactic disk population (PRP02). After we get the total number $N_{\text {Gal }}$ of a specific type of sources in the Galactic disk, then $\sim N_{\text {Gal }} / 100$ and $\sim N_{\text {Gal }} / 100 / 4$ sources of this population are expected to be found in Wang et al. and Muno et al. surveys, respectively, since the latter field contains stars 4 times less than the former (BT04). Note that sources found in these two surveys have different luminosity ranges.

\section{Results}

We adopt a variety of models (see Table 1), each with different assumptions for the parameters that govern the evolution in the calculations (common envelope parameter $\alpha$ and wind velocity parameter $\beta$ ). Tables 2 and 3 summarize the calculated numbers of various classes of X-ray sources contributed to Wang et al. and Muno et al. surveys, respectively. 
Table 1. Model parameters.

\begin{tabular}{cccc}
\hline Model & $\alpha$ & $\beta$ & tides \\
\hline $\mathrm{A}$ & 1.0 & 1.0 & OFF \\
$\mathrm{B}$ & 0.5 & 1.0 & OFF \\
$\mathrm{C}$ & 1.0 & 4.0 & OFF \\
$\mathrm{D}$ & 1.0 & 0.25 & OFF \\
$\mathrm{E}$ & 1.0 & 1.0 & ON \\
\hline
\end{tabular}

Table 2. The expected numbers of X-ray sources in Wang et al. survey with $L_{\mathrm{X}, 2-10 \mathrm{keV}}$ in the range of $10^{33}-10^{35} \mathrm{ergs}^{-1}$. In parentheses we list the expected numbers of X-ray binaries with WD donors. The expected numbers after correcting for the inclination effect are listed in the last column.

\begin{tabular}{cccccccc}
\hline Model & WNSs & NS LMXBs & BH LMXBs & IPs & Pulsars & TOTAL & TOTAL-inc \\
\hline A & $<1$ & $3(0)$ & $<1(0)$ & 1 & 9 & 13 & 13 \\
B & $<1$ & $<1(0)$ & $<1(0)$ & 1 & 9 & 9 & 9 \\
C & $<1$ & $4(0)$ & $<1(0)$ & 1 & 9 & 14 & 14 \\
D & $<1$ & $<1(0)$ & $<1(0)$ & 2 & 9 & 11 & 11 \\
E & $<1$ & $2(0)$ & $1(0)$ & 1 & 9 & 13 & 12 \\
\hline
\end{tabular}

Table 3. The expected numbers of X-ray sources in Muno et al. survey with $L_{\mathrm{X}, 2-10 \mathrm{keV}}$ in the range of $10^{30}-10^{33} \mathrm{ergs}^{-1}$.

\begin{tabular}{cccccccc}
\hline Model & IPs & NS LMXBs & BH LMXBs & Pulsars & Massive Stars & TOTAL & TOTAL-inc \\
\hline A & 165 & $648(646)$ & $132(0)$ & 28 & 72 & 1045 & 912 \\
B & 169 & $195(194)$ & $7(0)$ & 28 & 71 & 470 & 405 \\
C & 163 & $642(641)$ & $130(0)$ & 28 & 72 & 1035 & 905 \\
D & 176 & $935(934)$ & $158(0)$ & 28 & 72 & 1369 & 1202 \\
E & 706 & $1142(1141)$ & $270(0)$ & 28 & 72 & 2218 & 1963 \\
\hline
\end{tabular}

Figure 1 shows the total X-ray luminosity distributions for several models (results for models $\mathrm{C}$ and $\mathrm{D}$ share similar features as that in model A). Distributions after correcting for the inclination of accretion disks are also shown.

\section{Discussion and conclusions}

By using the EPS method, we have investigated the population of faint X-ray sources in the GCR. For Wang et al. survey, we find that a considerable fraction of the discrete sources are young pulsars and NS LMXB transients in quiescence, while WNSs proposed by PRP02 have negligible contribution due to the propeller effect. For Muno et al. field, IPs present a minor contribution, and the majority of the X-ray sources are NS LMXB transients with WD donor stars. The latter result is consistent with BT04 analysis.

The results are obviously subject to the star formation history and initial mass function (IMF) in the GCR. It is still a matter of debate as to whether the star formation is continuous or episodic, and whether it occurs only in localized regions or is relatively uniform throughout the GC (Muno et al. 2004). Figer et al. (2004) suggested that the stat formation is probably continuous over the last $\sim 10 \mathrm{Gyr}$, as we did in this paper. The molecular clouds in the GCR have higher densities and temperatures than those in the disk due to the tidal forces. This environment might favor the formation of massive stars (Morris 1993). The Hubble Space Telescope observation of Arches Cluster suggested 

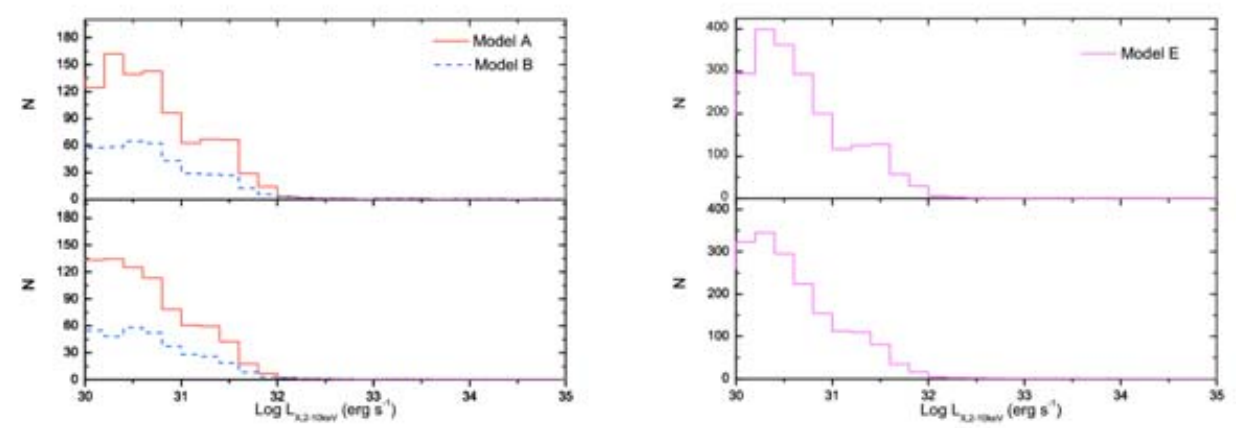

Figure 1. Left: The total number of X-ray sources as a function of X-ray luminosity for models A (solid lines) and B (dashed lines), in which no tidal effect is considered. The upper and lower panel show the intrinsic and apparent distributions after correcting for the accretion disk inclination effect respectively. Right: Luminosity function for model E with tidal effect considered.

a fairly flat IMF with a slope of $\sim-1.8$ (Figer et al. 1999). Portegies Zwart et al.(2002b), however, showed that the observed characteristics (unusually flat mass function and overabundance of massive stars) of the Arches cluster are consistent with a perfectly normal IMF. The observed anomalies are then caused by a combination of observational selection effects and the dynamical evolution of the cluster.

\section{Acknowledgements}

This work was supported by NSFC under grant number 10025314 and MSTC under grant number NKBRSF G19990754.

\section{References}

Belczynski, K., \& Taam, R. E. 2004, ApJ, 616, 1159 (BT04)

Chelbowski, T., \& Garmany, C. D. 1991, ApJ, 368, 241

Davies, R. E., \& Pringle, J. E. 1981, MNRAS, 196, 209

Figer, D. F. et al. 1999, ApJ, 525, 750

Figer, D. F. et al. 2004, ApJ, 601, 319

Hobbs G. et al. 2005, MNRAS, 360, 963

Hurley J. R. et al. 2000, MNRAS, 315, 543

Hurley J. R. et al. 2002, MNRAS, 329, 897

Ikhsanov, N. R. 2001, A\&A, 375, 944

Kube, J. et al. 2003, A\&A, 404, 1159

Menou, K., et al. 1999, ApJ, 520, 276

Morris, M. 1993, ApJ, 408, 496

Muno, M. P. et al. 2003, ApJ, 589, 225

Muno, M. P. et al. 2004, ApJ, 613, 1179

Pfahl, E. et al. 2002, ApJ, 571, L37 (PRP02)

Possenti, A. et al. 2002, A\&A, 387, 993

Portegies, Zwart, S. F. et al. 2002a, ApJ, 574, 762

Portegies, Zwart, S. F. et al. 2002b, ApJ, 565, 265

Seward, F. D., \& Wang, Z. 1988, ApJ, 332, 199

van Paradijs, J. 1996, ApJ, 464, L139

Wang, Q. D. et al. 2002, Nature, 415, 148

Zhang, S. N. 2005, ApJ, 618, L79 


\section{Discussion}

ERACLEOUS: What did you assume for the star formation history of the galactic center region?

LI: We assumed a constant SFR similar to the galactic disk.

LIPUNOV: What magnetic field decay hypothesis you used? The Kick velocity which you used 2 times high than reasonable value.

LI: We don't use any magnetic decay law. Only one, for HMBS - const $\sim 10^{12}$ Gs and for LMBS $-10^{8}$ Gs. 